\title{
Fast-Converging Algorithm for Wavefront Reconstruction based on a Sequence of Diffracted Intensity Images
}

\author{
Ni Chen, Jiwoon Yeom, Keehoon Hong, Gang Li, and Byoungho Lee* \\ School of Electrical Engineering, Seoul National University, Gwanak-gu, Gwanakro 1, \\ Seoul 151-744, Korea
}

(Received February 12, 2014 : revised April 15, 2014 : accepted April 23, 2014)

\begin{abstract}
A major advantage of wavefront reconstruction based on a series of diffracted intensity images using only single-beam illumination is the simplicity of setup. Here we propose a fast-converging algorithm for wavefront calculation using single-beam illumination. The captured intensity images are resampled to a series of intensity images, ranging from highest to lowest resampling; each resampled image has half the number of pixels as the previous one. Phase calculation at a lower resolution is used as the initial solution phase at a higher resolution. This corresponds to separately calculating the phase for the lower- and higher-frequency components. Iterations on the low-frequency components do not need to be performed on the higher-frequency components, thus making the convergence of the phase retrieval faster than with the conventional method. The principle is verified by both simulation and optical experiments.
\end{abstract}

Keywords: Phase retrieval, Wave-front sensing, Diffraction theory, Image reconstruction techniques OCIS codes : (100.5070) Phase retrieval; (010.7350) Wave-front sensing; (050.1960) Diffraction theory; (100.3010) Image reconstruction techniques

\section{INTRODUCTION}

Optical wavefronts that are reflected by or transmitted through an object experience amplitude and phase modulation by the object. If the light field is passing through an object, the measured wavefronts carry information related to the phase delay caused by the object, which is due to the refractive index and shape of the object; thus when the wavefront is measured, the thickness and shape of the object can be obtained. If the light field is reflected by an object, the wavefront carries information regarding the topology of the object, and thus can be used to measure the surface profile of the object. Hence wavefront measurements can be utilized in many areas, including imaging, surface profiling, adaptive optics, astronomy, ophthalmology, microscopy, and atomic physics [1,2]. However it is not possible to detect the phase using modern detectors because the oscillation frequency of light is too high to be followed by detectors. Therefore, many attempts to acquire phase information have been made in the past. The techniques for this can mainly be categorized into two types, one based on interferometry and the other a phase-retrieval method based on capturing the intensity of the object beam. The interferometry method, generally referred to as holography, records the interference pattern of an object beam and a reference beam. This process converts phase information to intensity modulation [3]. However, introducing a reference beam requires a complicated interference experimental setup, which also induces some other problems in hologram reconstruction, such as the DC-term problem and the twin-image problem. These problems require a more complicated experiment setup [4, 5].

Wavefront reconstruction using phase-retrieval techniques does not require any reference beam. Generally, several diffracted intensity images and some digital image postprocessing are needed. Deterministic phase retrieval using the transport of intensity equation (TIE) works under conditions of the paraxial approximation, which limits its application in general [6-8]. Iterative phase-retrieval methods mainly involve the use of the Gerchberg-Saxton (GS) and Yang$\mathrm{Gu}$ (YG) algorithms, which use prior knowledge of the object as constraints [9-11]. For the case of an object with

\footnotetext{
*Corresponding author: byoungho@snu.ac.kr

Color versions of one or more of the figures in this paper are available online.
} 
an unknown constraint, it is difficult to reconstruct the wavefront using the GS and YG algorithms. Various approaches have been proposed to solve this problem. One approach is to characterize the object indirectly, using an aperture to capture small images and then obtain the object constraint [12], or using a known illumination pattern as a constraint instead of the object constraint [13]. The other approach is to use two or more intensity images with variations, which can be produced by capturing intensity images with different focuses [14-16], translating an aperture transversely [17], or shifting the illumination [18]. However, all of these approaches require additional optical components and involve complicated digital postprocessing.

Pedrini et al. used images captured at axial translated planes, i.e., single-beam multiple-intensity reconstruction (SBMR) [19]. The SBMR method has the simplest capture setup compared to the other phase-retrieval methods based on multiple intensity images [14-18]: It uses only one camera. It has also been applied in some other research areas, such as measuring the shape, deformation, and angular displacement of a three-dimensional object $[20,21]$. Phase retrieval using multiple intensity images captured at a series of planes can detect spatial frequencies at different sensitivities. Therefore, the significant amount of data carried by these intensity images makes this approach very robust and rather stable to the effects of noise [22]. Figure 1 shows the experimental scheme for capturing intensity images by the SBMR method. The object is illuminated by a collimated light source. The charge-coupled device (CCD) is shifted along the optical axis and captures the diffracted intensity images with an interval $\Delta z$ between adjacent images. The total number of intensity images is $K$.

It is known that the number of captured images affects reconstruction quality, with more intensity images resulting in higher-quality reconstructions [23]. However, capturing more intensity images requires more movement steps of the camera or the object, which makes the captured images sensitive to small misalignments in the experimental setup, and thus the noise in the captured intensity images becomes more serious. Furthermore, capturing more intensity images requires more experimental time, which makes the method not suitable for dynamic-object or real-time applications. In order to improve the capabilities of SBMR [19], beam splitters [24], spatial light modulators (SLM) [25, 26], or deformable mirrors (DM) [27] were used to achieve single-

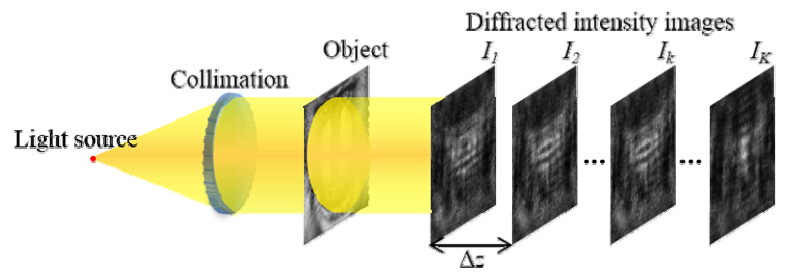

FIG. 1. Capturing process for the speckle intensity images for SBMR. shot or single-plane intensity image capturing processes. However, beam splitters cause the attenuation of illuminating light. In the cases of SLM and DM, the simplicity of the experimental setup is sacrificed. A random amplitude mask [28] and random phase plate [29] have also been used to transform low frequency components to fast varying high frequencies, which requires fewer iterations for reconstruction. However, an amplitude mask results in the diminution of light energy, and the use of a phase plate requires difficult fabrication.

Multiscale signal representation is a very efficient tool that can be used in signal and image processing [30, 31]. It represents signals or images with different resolutions: The high-resolution images carry the high spatial frequencies of the original image, and the low-resolution images carry the low spatial frequencies. An example is shown in Fig. 2. Figure 2(a) shows an image with 128 pixels and Fig. 2(b) shows a scaled image with 64 pixels, which looks like a low-pass-filtered image of Fig. 2(a), i.e. Fig. 2(b) contains the low frequencies of the image [32]. In the conventional phase-retrieval method, because the high-frequency components of the object vary faster than the low-frequency components, the variation between two diffracted intensity images is larger for the higher-frequency components of the object than the low-frequency components of the object. Therefore, fewer iterations are needed to recover the high-frequency than the low-frequency components. This is why the convergence proceeds rapidly in the beginning, and then becomes very slow.

In this study, we optimized the SBMR method by using a multi-scale technique. The captured diffractive intensity images are resampled to several images with different resolutions. Phase retrieval is first performed on the images resampled with a lower resolution, and then transferred to images that are resampled with a higher resolution. This corresponds to performing more iterations for the low-frequency components than the high-frequency components. Suppose a captured image is resampled to some number $A$ of level images, and the number of iterations at each level is $B$. Then for the low-frequency components of the lowestresolution image the number of iterations would be $A B$,

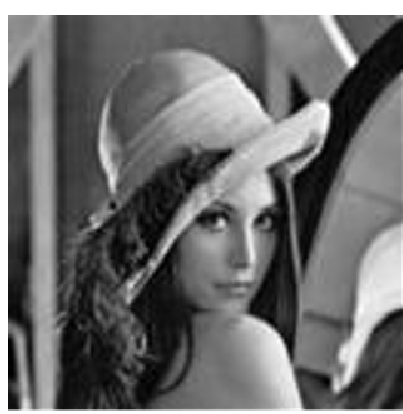

(a)

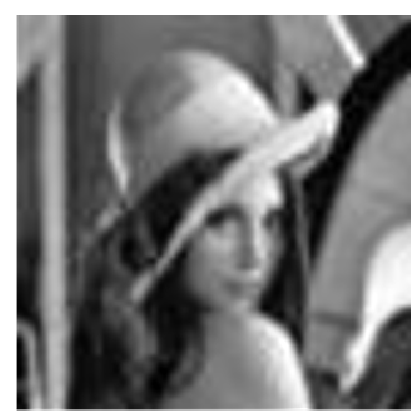

(b)
FIG. 2. Example of resampling an image (a) with 128 sampling points and (b) with 64 sampling points. 
and for the high-frequency components of the highestresolution image the number of iterations would be just $B$. Moreover, because the image that is resampled with a low resolution contains fewer pixels, the time required for one iteration for this image is less than that for an image resampled at a higher resolution. Because the image resampling process corresponds to separating the high-frequency components from the low-frequency components, different numbers of iterations can be performed for low-frequency components and high-frequency components separately. Therefore, although we perform more iterations for the images resampled with low resolutions than for images resampled with high resolutions, the total time consumed does not increase by as much as in the conventional method. Furthermore, the large variation between intensity images results in fast convergence of the iteration process [33], and the variation between images that are resampled with low resolution is increased. For these reasons, the proposed algorithm achieves a faster convergence than the conventional method. We describe the principle of the proposed method in Section 2, and the results of computer simulations and experiments to verify the proposed method are shown in Sections 3 and 4.

\section{PRINCIPLE}

In our proposed algorithm the capturing process is the same as that for the conventional method, as shown in Fig. 1. A series of speckle diffractive intensity images $I_{k}$ is captured along the optical axis at different positions, where $k$ is an integer from 1 to $K$. When the captured images have a lateral resolution of $2^{M}$ pixels, the captured intensity image can be resampled into $M$ different level images. Therefore, the resampled image of the $m^{\text {th }}$ level has $2^{m}$ sampling points, where $m$ is an integer from 1 to $M$. The resampling process starts from the captured image which has the most sampling points, i.e., where $I_{k}^{m}=I_{k}^{M}$. The next sampling level $I_{k}^{m-1}$ can be expressed as

$$
I_{k}^{m-1}=Z\left\{I_{k}^{m}, 2^{m-1}\right\}
$$

where $Z$ is the bicubic interpolation, in which the value of the pixel that needs to be calculated is determined by the average value of its 16 weighted neighboring pixels. The weight of each neighboring pixel is calculated using 16 equations, which are given by the gradients in both the horizontal and vertical directions, and the cross derivatives at each of the four corners of the pixel square [34, 35]. The second parameter of the $Z$ operation is the image size after performing an interpolation. This process is performed until the resampling level reaches the first resampling level $I_{k}^{1}$. The whole resampling process of $I_{k}$ is performed for all of the captured intensity images $I_{1} \cdots I_{K}$. After this resampling process we have a total of $M \times K$ intensity images, and these images are grouped by resampling level. For example, the $m^{\text {th }}$ group of the resampled intensity images is $G_{m}=\left\{I_{1}^{m}, I_{2}^{m}, \cdots I_{K}^{m}\right\}$. The bicubic interpolation is used to preserve the fine detail with better performance than other common interpolation algorithms, such as bilinear interpolation or nearest-neighbor interpolation [34, 35]. The 'sinc' interpolation may be thought to be the most appropriate method for band-limited optical fields, but oscillations at signal borders may restrict image processing. Particularly in small images, noticeable ripples from the borders may occupy a substantial part of the image [3638]. Considering the reduced size of the resampled images in our approach, i.e. $2^{1}$ to $2^{N}$ pixels, sinc interpolation is not appropriate in our method. Figure 3 shows an example of the resampling scheme for an image that has $2^{4}$ pixels.

Figure 4 shows the flowchart for our proposed algorithm. The circled numbers in this figure denote the steps of the procedure. In the flowchart, $N$ is the number of iterations,

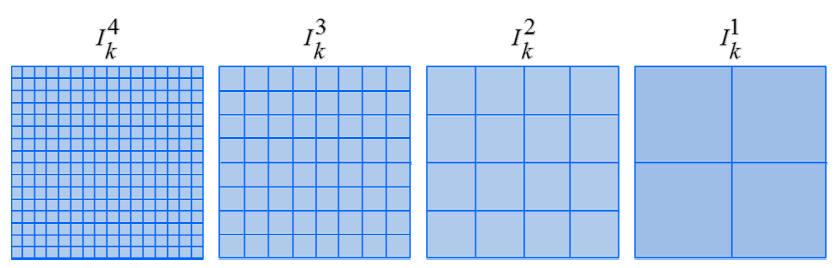

FIG. 3. Example of the resampling process for an image with $M=4$.

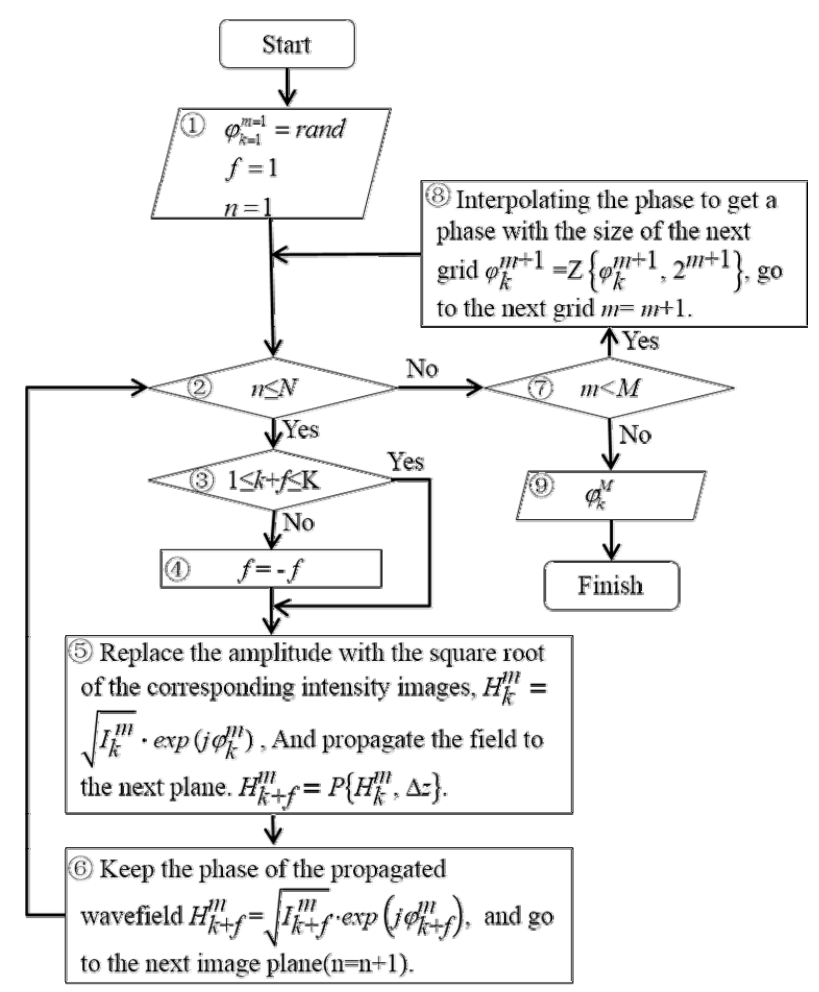

FIG. 4. Flow chart of the proposed algorithm. 
and $f$ is a binary value of 1 or -1 , where 1 denotes forward propagation and -1 denotes backward propagation. The conventional iteration method is performed from the first resampling level, i.e. $M=1$ and $G_{1}=\left\{I_{1}^{1}, I_{2}^{1}, \cdots I_{K}^{1}\right\}$. The solution at this level is interpolated to the next resampling level $(M=2)$ and used as the initial solution for the next resampling level. This process is continued until the highest resampling level $G_{M}$ is reached. It should be noted that this is different from optical ptychography, which simply uses a part of the intensity images and extends the image areas until the full intensity images are addressed [39].

The details of the iterative procedure are as follows.

Step 1: Set the initial value. The procedure starts from the first image at the first level, i.e. $k=1$ and $m=1$. The initial complex field is a composite of an amplitude and a random initial phase. The square root of the intensity $I_{1}^{1}$ is used as the amplitude, and the initial phase is a random distribution that has $2^{1}$ pixels, which is the size of the lowest sampled level.

Step 2: Check the current iteration number. When the iteration number reaches the maximum iteration value, go to the next level, i.e. go to step 7; otherwise, perform an iteration at the current level, i.e. go to step 3 .

Step 3: Decide which process should be continued according to whether the current image is the last, the first, or not in the current group. If not, do the forward propagation to the next image, i.e. go to step 5; otherwise, do the backward propagation, i.e. go to step 4 .

Step 4: Reverse the propagation direction.

Step 5: Build the complex wave field. Use the square root of the current intensity distribution as the amplitude, and the previously calculated phase as the phase: $H_{k}^{n}=$ $\left(I_{k}^{m}\right)^{1 / 2} \cdot \exp \left(j \varphi_{k}^{m}\right)$. Then propagate this wavefield to the next plane: $H_{k+f}^{n}=P\left\{H_{k}^{n}, \Delta \mathrm{z}\right\}$. Here $P$ indicates the angular spectrum propagation of the Rayleigh-Sommerfeld diffraction [40]:

$$
\begin{aligned}
& P\{O(x, y), z\} \\
& =\mathfrak{J}^{-1}\left(\mathfrak{I}[O(x, y)] \times \exp \left[-j \frac{2 \pi z}{\lambda} \sqrt{1-\lambda^{2} u^{2}-\lambda^{2} v^{2}}\right],\right.
\end{aligned}
$$

where $J$ is the operation of the Fourier transform, $\lambda$ is the illumination wavelength, and $(u, v)$ are the coordinates after propagation.

Step 6: Updating the amplitude of the calculated complex wavefront $H_{k+f}^{n}$ in the next plane by using the square root of the intensity image at this plane: $H_{k f f}^{n}=\left(I_{k+f}^{n}\right)^{1 / 2} \cdot \exp \left(j \varphi_{k+f}^{m}\right)$. Also update the index of the iteration and intensity image. Shift the process to the next image plane, i.e. go to step 2 .

Step 7: If the current level is the last level, retain the calculated phase in step 6 as the final solution. Otherwise, using the solution in step 6 as the initial phase of the next level, go to step 8 .

Step 8: Interpolate the calculated phase in step 6 to the size of the next level using Eq. (1). Here the second parameter in this equation should be $2^{m+1}$. Perform steps 2 to step 8 until the iteration number that was set previously is reached.

From the final calculated wave field, the wavefront at all the captured image planes can be obtained by propagating this field, and the object can be reconstructed by back-propagating the wavefront to any image plane.

As presented in the introduction, the images sampled with fewer points carry the low-frequency components of the image, which requires fewer iterations in the phase reconstruction, and vice versa. This is indicated in Fig. 5. The two images with 128 and 64 pixels in Figs. 2(a) and 2(b) are reconstructed using the conventional error-reduction method. The normalized root-mean-square errors (RMSEs) according to the iteration number are plotted. The red dashed and blue solid lines indicate the convergence of the iterations for Figs. 2(a) and 2(b), respectively. It can be seen that the iterative convergence for the image sampled with 64 pixels is faster than for that with 128 sampling points.

Resampling the images also produces increased variations

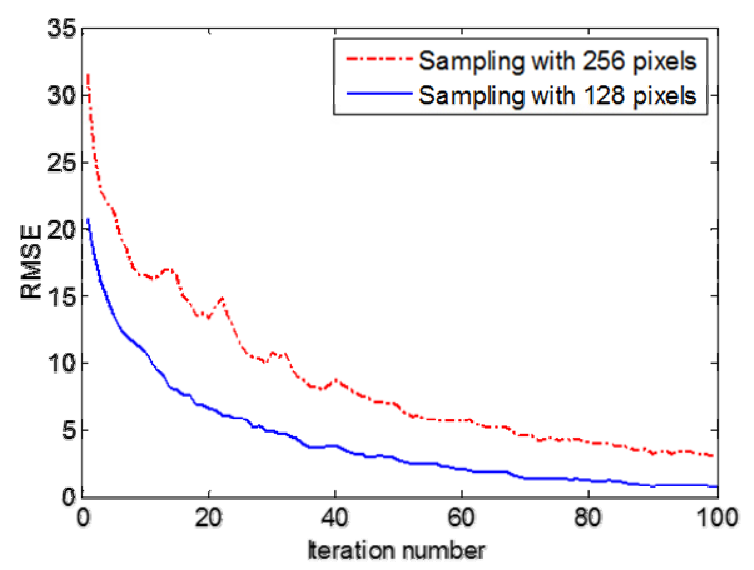

FIG. 5. Iterative convergence for images with different sampling points.

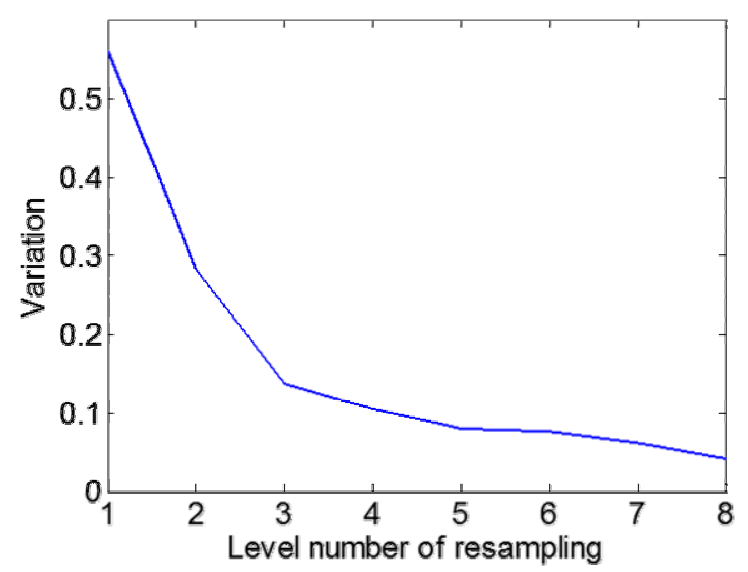

FIG. 6. Pixel variation between two diffracted images along the optical axis at each resampling level. 
between images. Figure 6 shows an example of the variations between diffracted images along the optical axis with varying resampling levels. Figure 2(a) is used to generate two diffracted images with a slice-depth difference. The two images are resampled to 8 levels respectively. The variations for different level images are then calculated. The horizontal axis represents the level number and the vertical axis represents the variations with the corresponding sampling level on the horizontal axis. This figure demonstrates that the variation between images at two planes along the optical axis increases with decreasing sampling numbers, which is the requirement for achieving fast convergence in iterative phase-retrieval techniques. This can be attributed to the fast convergence of phase retrieval because the amount of defocusing between the diffracted intensity images affects the accuracy of the phase retrieval [41]. The amount of defocusing can be obtained with a larger depth difference, in our method, by just carrying out a post-resampling of the diffracted images.

Based on the two points presented above, we can speculate that resampling diffracted intensity images with different levels makes it possible to perform a fasterconverging iterative phase retrieval.

\section{NUMERICAL SIMULATIONS}

In the simulation, the object is a composition of the amplitude pattern shown in Fig. 7(a) and a random phase distribution ranging from 0 to $2 \pi$. The size of the object is $256 \times 256$ pixels. The object is padded with zeroes to avoid energy leakage from the edges of the captured intensity images. In experiment, this corresponds to making almost all of the intensity distribution be within the camera sensor area. The object is illuminated by a light source with a wavelength of $532 \mathrm{~nm}$. Five intensity images are captured by a CCD camera with a pixel pitch of $4.65 \mu \mathrm{m}$. The nearest capture plane is located at a distance from the object plane of $z=30 \mathrm{~mm}$, and the interval between two adjacent capture planes is $\Delta z=5 \mathrm{~mm}$. Figure $7(\mathrm{~b})$ is the calculated diffracted intensity image at the first plane, and Fig. 7(c) shows eight resampled images of the first image $I_{1}$.

The original image is reconstructed by both the conventional and the proposed methods, respectively. The normalized RMSE is used to measure the results. Figure 8 shows the RMSE with respect to time consumption. The proposed and conventional reconstructions are plotted as a red dashed line and a blue solid line, respectively. Figure $8(\mathrm{~b})$ is the square area crossed by the blue dashed line in Fig. 8(a). From Fig. 8(b) it can be seen that the conventional method reached a minimum RMSE value at about 4.5 seconds, while the proposed method arrived at the same RMSE value in about 2.2 seconds, which is about $51 \%$ faster than the conventional method.

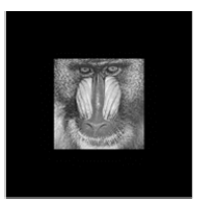

(a)
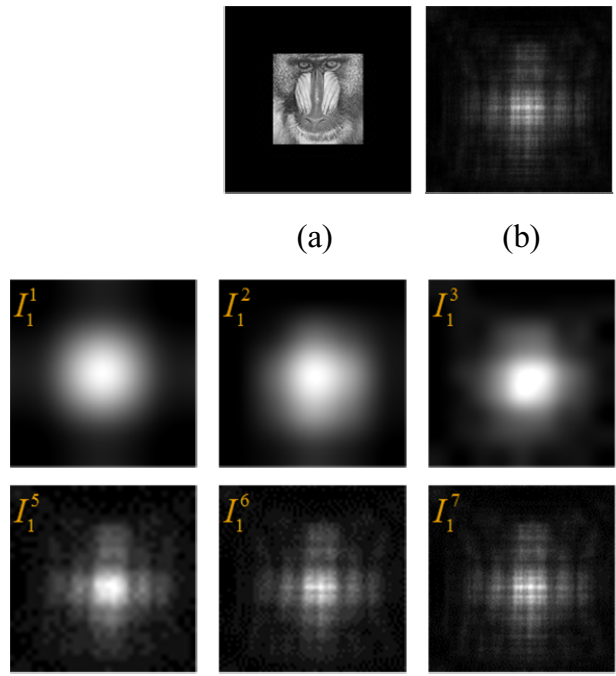

(b)
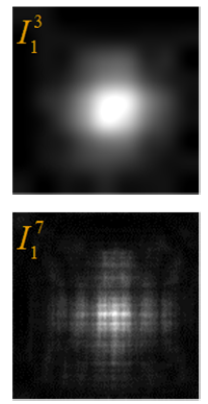

(c)

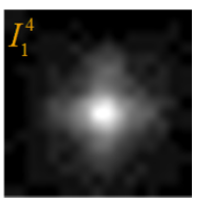

FIG. 7. Example of the resampling of an intensity image: (a) Amplitude image of the object used in the numerical simulation, (b) the first captured intensity image and (c) resampled images.

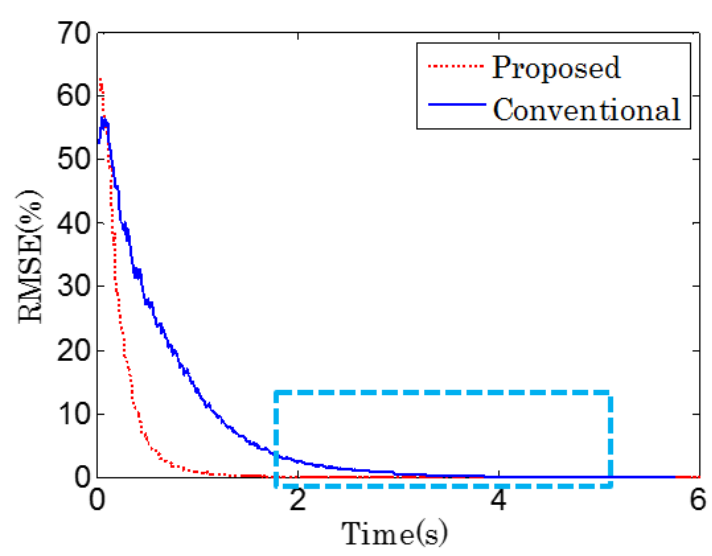

(a)

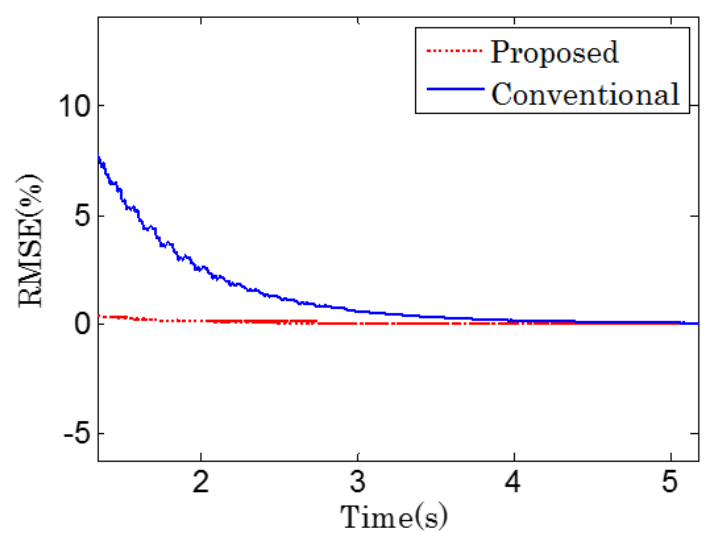

(b)

FIG. 8. RMSE with respect to time consumption. Part (b) is the magnification of the square area crossed by blue lines in part (a). 


\section{EXPERIMENTAL RESULTS}

In the experiment, a laser with a wavelength of $532 \mathrm{~nm}$ was used to illuminate a Newport USAF 1951 resolution chart. In the resolution chart the background area is opaque, while the line area is transparent. The light wave after the object is modulated in the line area and stopped in the opaque area. Thus the optical wavefront, just after the object, reflects the shape of the resolution chart. By reconstructing the wavefront at the location of the object, the shape image of the object can be reconstructed. Seven diffracted intensity images were captured by a Point Grey FL2-14S3M/C CCD camera. The first image was located a distance of $40 \mathrm{~mm}$ from the target, and the interval between the two images was $8 \mathrm{~mm}$. Figure 9 shows the experimental setup. Figure 10(a) shows the directly captured image of the resolution chart, while Figs. 10(b)-(h) show the captured diffracted intensity images. The wavefront at the first plane was calculated by both the conventional and the proposed methods, respectively. The object was reconstructed by digitally propagating the calculated wavefront.

Three hundred iterations were performed in both the conventional and proposed methods. The corresponding time consumption is shown in Fig. 11. The horizontal dashed lines are the chosen timelines for convergence comparison. The red dashed line and blue solid line represent the

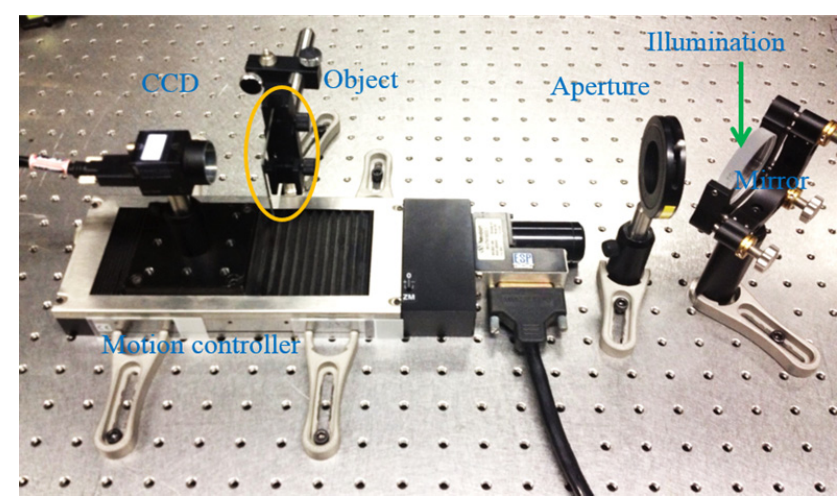

FIG. 9. Experiment setup.

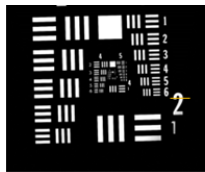

(a)

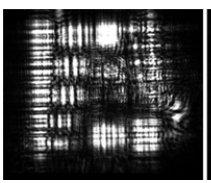

(e) $\mathrm{z}=64 \mathrm{~mm}$

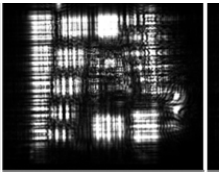

(b) $\mathrm{z}=40 \mathrm{~mm}$

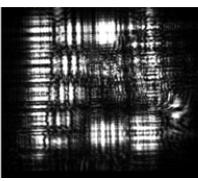

(f) $\mathrm{z}=70 \mathrm{~mm}$

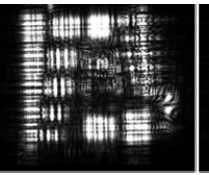

(c) $\mathrm{z}=48 \mathrm{~mm}$

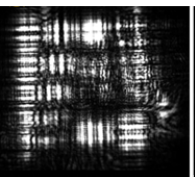

(g) $\mathrm{z}=78 \mathrm{~mm}$

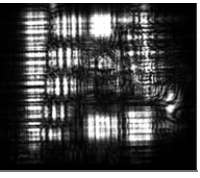

(d) $\mathrm{z}=56 \mathrm{~mm}$
FIG. 10. Captured images: (a) the directly captured image of the object; (b)-(h) the captured diffracted intensity images. proposed and conventional methods, respectively. The horizontal axis values of the two intersection points on each horizontal dashed line indicate the numbers of iterations used in the conventional and proposed methods, respectively, and are listed in Table 1 . The corresponding reconstructed images for the iteration numbers in Table 1 are shown in Fig. 12. The lines crossing the "2" character

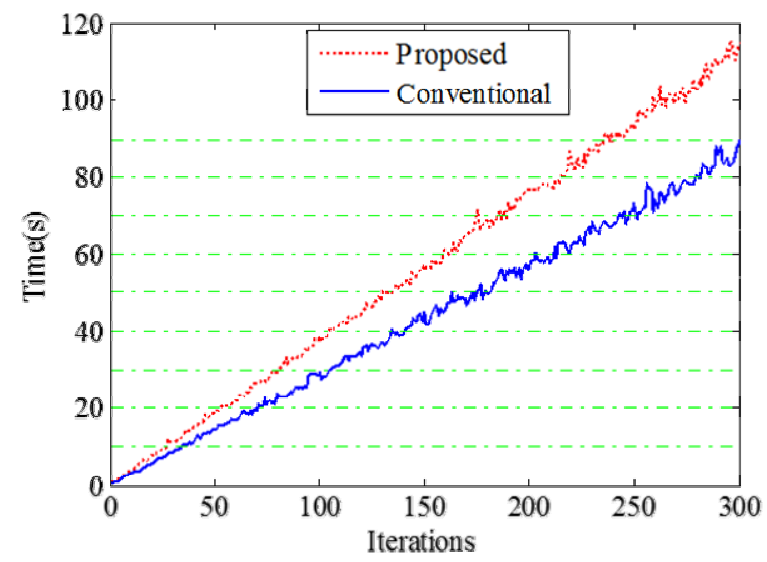

FIG. 11. Time consumption versus the number of iterations.

TABLE 1. Iteration requirements for the crossed points in Fig. 11

\begin{tabular}{c|c|c|c|c|c|c|c|c}
\hline $\begin{array}{c}\text { Time(s) } \\
\text { Iterations }\end{array}$ & 10 & 20 & 30 & 40 & 50 & 60 & 70 & 80 \\
\hline Conventional & 35 & 75 & 106 & 141 & 173 & 203 & 243 & 280 \\
\hline Proposed & 27 & 53 & 82 & 105 & 133 & 159 & 186 & 215 \\
\hline
\end{tabular}

$\mathrm{t}=10 \mathrm{~s}$

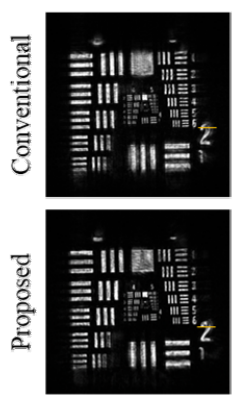

$\mathrm{t}=50 \mathrm{~s}$
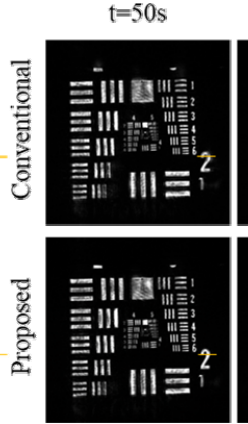

$\mathrm{t}=20 \mathrm{~s}$
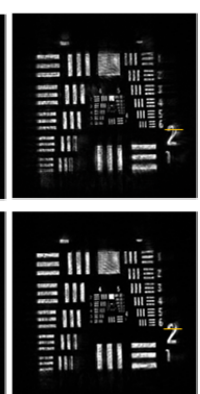

$\mathrm{t}=60 \mathrm{~s}$
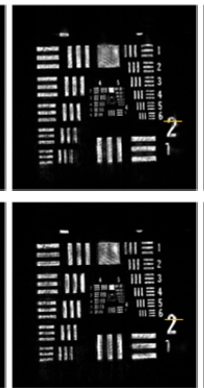

$\mathrm{t}=30 \mathrm{~s}$
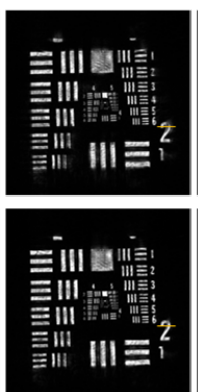

$\mathrm{t}=70 \mathrm{~s}$

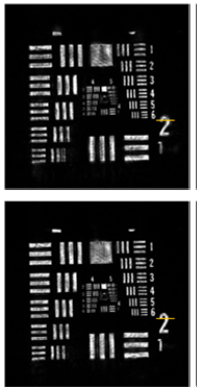

$\mathrm{t}=40 \mathrm{~s}$

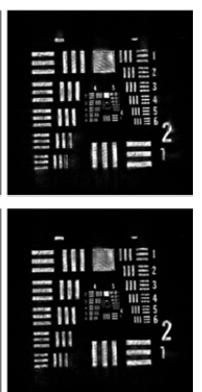

$\mathrm{t}=80 \mathrm{~s}$

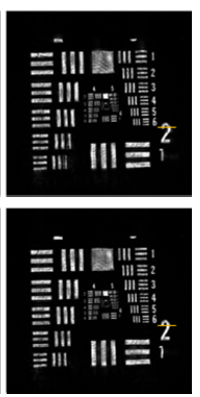

FIG. 12. The conventional and proposed reconstructions at the timelines. 


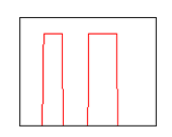

(a)
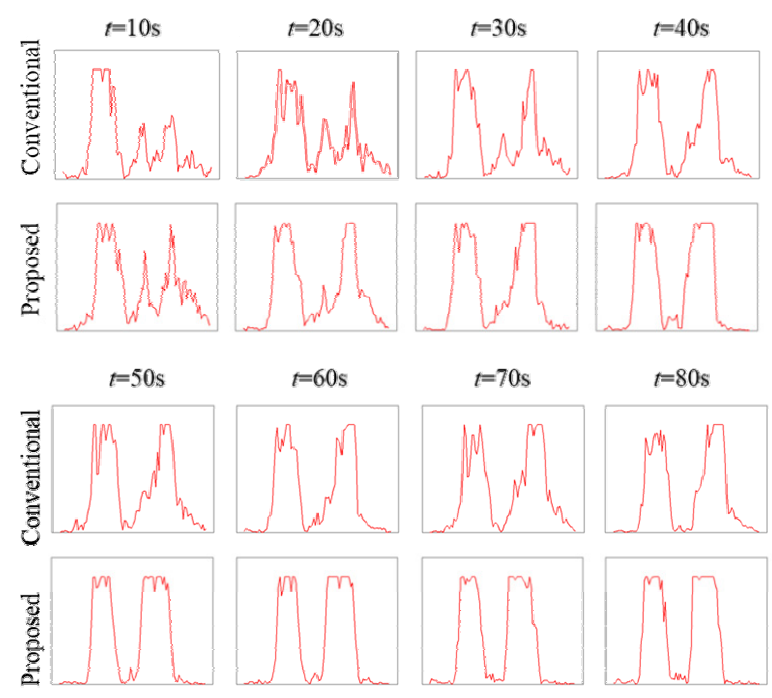

(b)

FIG. 13. The plotted images along the lines (a) on the direct captured image of Fig. 10(a) and (b) on the reconstructed images of Fig. 12.

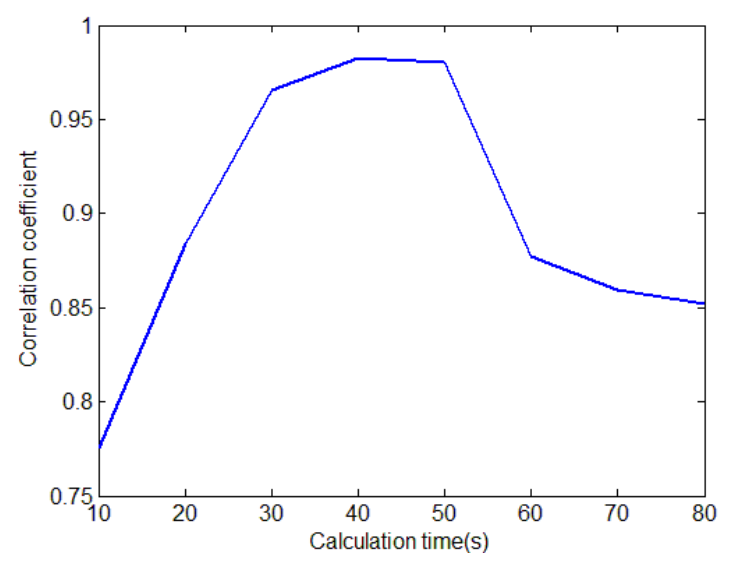

FIG. 14. The correlation coefficients between each proposed reconstructed image and the conventional image reconstructed with a time consumption of 40 seconds.

are plotted in Fig. 13(b). Figure 13(a) shows a plot corresponding to the directly captured object, i.e. Fig. 10(a), which is used as the reference. From Fig. 13 it can be seen that, in the conventional method, about 80 seconds were needed to obtain the best reconstruction. In order to find how much time is needed for the proposed method to reconstruct the image with a quality similar to this image, we compared this image with the proposed reconstructed images for time consumptions of $10 \mathrm{~s}, 20 \mathrm{~s}, 30 \mathrm{~s}, 40 \mathrm{~s}, 50 \mathrm{~s}$, $60 \mathrm{~s}, 70 \mathrm{~s}$, and $80 \mathrm{~s}$. The correlation coefficients are plotted as Fig. 14. From this figure, a similar image was obtained with the proposed method within 40 seconds. This means that the proposed method is capable of obtaining a similar reconstruction to the original with a time consumption of about 40 seconds, which is about $50 \%$ faster than the conventional method with the case of our example images. This result is also in agreement with the simulated results. The small stagnation in the reconstructions by the proposed method is induced by the noise in the experimental images.

\section{CONCLUSION}

We propose a fast-convergening wavefront reconstruction algorithm by resampling diffractive intensity images. Both simulation and experimental results show that the convergence of the proposed method is about $50 \%$ faster than the conventional method for the case of our test images. We expect that the proposed method can be applied to realtime phase-retrieval applications, with further development of computer processor speeds.

\section{ACKNOWLEDGMENT}

This work was supported by the IT R\&D program of MSIP/KEIT (fundamental technology development for digital holographic contents).

\section{REFERENCES}

1. W.-D. Joo, "Wavefront sensitivity analysis using global wavefront aberration in an unobscured optical system," J. Opt. Soc. Korea 16, 228-235 (2012).

2. C. M. Vest, Holographic Interferometry (Wiley, New York, USA, 1979).

3. D. Gabor, "A new microscopic principle," Nature 161, 777778 (1948).

4. E. N. Leith and J. Upatnieks, "Wavefront reconstruction with continues-tone objects," J. Opt. Soc. Am. 53, 13771381 (1963).

5. T. Kreis and W. Juptner, "Suppression of the dc term in digital holography," Opt. Eng. 36, 2357-2360 (1997).

6. M. R. Teague, "Irradiance moments: Their propagation and use for unique retrieval of phase," J. Opt. Soc. Am. 72, 1199-1209 (1982).

7. T. E. Gureyev, A. Roberts, and K. A. Nugenr, "Partially coherent fields, the transport of intensity equation, and the phase uniqueness," J. Opt. Soc. Am. A 12, 1942-1946 (1995).

8. D. Paganin and K. A. Nugent, "Noninterferometric phase imaging with partially coherent light," Phys. Rev. Lett. 80, 2586-2589 (1998).

9. R. W. Gerchberg and W. O. Saxton, "A practical algorithm for the determination of phase from image and diffraction plane pictures," Optik 35, 227-246 (1972).

10. J. R. Fienup, "Phase retrieval algorithms: A comparison," Appl. Opt. 21, 2758-2769 (1982). 
11. G. Z. Yang, B. Z. Dong, B. Y. Gu, J. Y. Zhuang, and O. K. Ersoy, "Gerchberg-Saxton and Yang-Gu algorithms for phase retrieval in a nonunitary transform system: A comparison," Appl. Opt. 33, 209-218 (1994).

12. J. R. Fienup and A. M. Kowalczyk, "Phase retrieval for a complex-valued object by using a low-resolution image," J. Opt. Soc. Am. A 7, 450-458 (1990).

13. J. R. Fienup, "Lensless coherent imaging by phase retrieval with an illumination pattern constraint," Opt. Express 14, 498-508 (2006).

14. W. O. Saxton, "Correction of artefacts in linear and nonlinear high resolution electron micrographs," J. Microsc. Spectrosc. Electron. 5, 661-670 (1980).

15. D. L. Misell, "A method for the solution of the phase problem in electron microscopy," J. Phys. D: Appl. Phys. 6, L6-L9 (1973).

16. D. L. Misell, "An examination of an iterative method for the solution of the phase problem in optics and electron optics," J. Phys. D: Appl. Phys. 6, 2200-2225 (1973).

17. G. R. Brady, M. Guizar-Sicairos, and J. R. Fienup, "Optical wavefront measurement using phase retrieval with transverse translation diversity," Opt. Express 17, 624-639 (2009).

18. J. M. Rodenburg and H. M. L. Faulkner, "A phase retrieval algorithm for shifting illumination," Appl. Phys. Lett. 85, 4795-4797 (2004).

19. G. Pedrini, W. Osten, and Y. Zhang, "Wave-front reconstruction from a sequence of iterferograms recorded at different planes," Opt. Lett. 30, 833-835 (2005).

20. A. Anand, V. K. Chhaniwal, P. Almoro, G. Pedrini, and W. Osten, "Shape and deformation measurements of 3D objects using volume speckle field and phase retrieval," Opt. Lett. 34, 1522-1524 (2009).

21. P. F. Almoro, G. Pedrini, A. Anand, W. Osten, and S. G. Hanson, "Angular displacement and deformation analyses using a speckle-based wavefront sensor," Appl. Opt. 48, 932-940 (2009).

22. K. A. Nugent, "X-ray noninterferometric phase imaging: A unified picture," J. Opt. Soc. Am. A 24, 536-547 (2007).

23. P. Almoro, G. Pedrini, and W. Osten, "Complete wavefront reconstruction using sequential intensity measurements of a volume speckle field," Appl. Opt. 45, 8596-8605 (2006).

24. P. Almoro, A. M. S. Maallo, and S. G. Hanson, "Fastconvergent algorithm for speckle-based phase retrieval and a design for dynamic wavefront sensing," Appl. Opt. 48, 1485-1493 (2009).

25. L. Camacho, V. Micó, Z. Zalevsky, and J. García, "Quantitative phase microscopy using defocusing by means of a spatial light modulator," Opt. Express 18, 6755-6766 (2010).

26. A. Agour, P. F. Almoro, and C. Falldorf, "Investigation of smooth wave fronts using SLM-based phase retrieval and a phase diffuser," J. Eur. Opt. Soc.-Rapid 7, 12046 (2012).

27. P. Almoro, J. Glückstad, and S. G. Hanson, "Single-plane multiple speckle pattern phase retrieval using a deformable mirror," Opt. Express 18, 19304-19313 (2010).

28. A. Anand, G. Pedrini, W. Osten, and P. Almoro, "Wavefront sensing with random amplitude mask and phase retrieval," Opt. Lett. 32, 1584-1586 (2007).

29. P. F. Almoro and S. G. Hanson, "Random phase plate for wavefront sensing via phase retrieval and a volume speckle field," Appl. Opt. 47, 2979-2987 (2008).

30. J. L. Crowley and A. C. Sanderson, "Multiple resolution representation and probabilistic matching of 2-D gray-scale shape," IEEE Trans. Pattern Anal. Mach. Intel. 9, 113-121 (1987).

31. T. Lindeberg, "Scale-space for discrete signals," IEEE Trans. Pattern Anal. Mach. Intel. 12, 234-254 (1990).

32. B. E. A. Saleh and M. C. Teich, Fundamental of Photonics, $2^{\text {nd }}$ ed. (John Wiley \& Sons, Inc., Hoboken, United States, 2007).

33. M. R. Teague, "Irradiance moments: Their propagation and use for unique retrieval of phase," J. Opt. Soc. Am. 72, 1199-1209 (1982).

34. T. Acharya and P. S. Tsai, "Computational foundations of image interpolation algorithms," ACM Ubiquity 8, page 4 (2007).

35. F. P. Miller, A. F. Vandome, and J. McBrewster, Bicubic Interpolation (Alphascript Publishing, United States, 2010).

36. D. Fraser, "Interpolation by the FFT revisited - An experimental investigation," IEEE Trans. Acoust. Speech Signal Process 37, 665-675 (1989).

37. T. Smith, M. S. Smith, and S. T. Nichols, "Efficient sinc function interpolation technique for center padded data," IEEE Trans. Acoust. Speech Signal Process 38, 1512-1517 (1990).

38. L. Yaroslavsky, "Efficient algorithm for discrete sinc interpolation," Appl. Opt. 36, 460-463 (1997).

39. A. M. Maiden, J. M. Rodenburg, and M. J. Humphry, "Optical ptychography: A practical implementation with useful resolution," Opt. Lett. 35, 2585-2587 (2010).

40. K. Matsushima and T. Shimobaba, "Band-limited angular spectrum method for numerical simulation of free-space propagation in far and near fields," Opt. Express 17, 1966219673 (2009).

41. S. Mayo, P. Miller, S. Wilkins, T. Davis, D. Gao, T. Gureyev, D. Paganin, D. Parry, A. Pogany, and A. Stevenson, "Diversity selection for phase-diverse phase retrieval," J. Microscopy 207, 79-96 (2002). 